\title{
Out-of-step Protection combining Three-impedance Element Model with the Equal Area Criterion
}

\author{
Liang Yuan ${ }^{1, a}$, Zhaorong Zhang ${ }^{1, b}$ and Chong Chen ${ }^{1, c}$ \\ ${ }^{1} 200$ Xiao Ling Wei Street, Nanjing, Jiangsu, China \\ ayuanliangabc@126.coml, 'bhaorong0821@163.com, 'chenchong1994@126.com
}

\begin{abstract}
Keywords: three-impedance element model, equal area criterion, out-of-step protection, PSCAD simulation
\end{abstract}

Abstract This paper proposes a new procedure for out-of-step protection through the combination of a three-impedance element model with equal area criterion. The $P-\delta$ curve of the system is transferred into $P-t$ curve and then $P-t$ curve is applied to forecast whether the oscillation between the generator and the power system is out-of-step or stable. The forecasting results determine the operation of the out-of-step protection: If the oscillation is unstable, the signals will be sent during the first period of unstable oscillation, and then the number of sliding pole will be counted to achieve a delay action. Otherwise, no response will be made. A three-machine infinite bus system has been used to test the proposed method by simulation with PSCAD software. The simulations test the protection in a variety of faults within the system, including unstable and stable oscillation, loss of excitation and short-circuit faults. Simulation results confirm that this method can work effectively as a backup protection when faults such as loss of excitation and short-circuit fault are not promptly removed. Moreover, the simulations provide a reliable basis for the scheme and design of out-of-step protection in the power plant.

\section{Introduction}

Electromechanical oscillation in power systems occurs due to an imbalance between input and output powers. Whether the power system will return to a stable state depends on the level of the disturbance. The oscillations will not damp out after a severe disturbance and consequently lead to an unstable operating condition called an out-of-step condition. In order to lead the rest of the system back into a new stable condition, the out-of-step relays are equipped to detect such conditions in the power system and disconnect part of the system at preselected locations [1].

Currents and voltages oscillate in amplitude and phase during power system swings. At certain locations of the power system, the calculated impedances will be so small during a disturbance that they enter the protection dead zones of distance relays [2]. In many cases where the disturbance is not severe, the systems should be allowed to settle down to a stable state. In other cases, the systems should be disconnected from one another as they are likely to become unstable. After disconnection, the individual systems should be allowed to settle down to stable states. In order to maintain generation load balance, it at times become necessary to shed load or generation, which depends on the situation of the disturbance. Conventional power swing relays use distance relays and delay timers to distinguish power swings that are likely to result in system instability from those which are likely to settle into stable states [3].

There are various techniques available in literature and in practice to detect out-of-step conditions. The most popular and well-known out-of-step detection technique in China is the three-impedance element model based on the variation of apparent impedance locus at machine terminal. When applied to multi-machine systems, it can distinguish whether the oscillation center is internal or external of the generator-transformers [4]. This method can also adjust setting valves of allowable tripping current and the number of sliding pole. Thus, the three-impedance element model can meet the basic requirements of out-of-step protection. However, because of the use of time relay and D flip-flop, the technique is unable to forecast swings that are likely to result in dynamic instability, which means that from the point of view of avoiding loss-of-synchronism, it is too late to take suppression actions like shedding load or generation [5]. 
In [6] an out-of-step detection technique based on the classical equal area criterion (EAC) in the power angle domain was proposed. The EAC in $\delta$ domain has an advantage over the other techniques in which it does not need any offline calculations and system analysis. However this method required pre- and post-disturbance P- $\delta$ curves of the system. As the P- $\delta$ curves are dependent on the system parameters, many measurement and communication devices at various locations are required to gather the current system information [7].

On the basis of three-impedance element model, this paper uses the concept of EAC modified to the time domain. Thus, an out-of-step protection methodology is proposed taking the advantage of time domain EAC to predict the possibility of out-of-step condition. The time domain EAC is based on the P-t curve instead of the P- $\delta$ curves. The combination of the three-impedance element model with the EAC constitutes the proposed protection. Firstly, the out-of-step protection based on the EAC targets at signals, predicting the potential out-of-step conditions and taking suppression actions (shedding load or generation, taking electric braking and etc.). Secondly, the three-impedance element model targets at generator tripping and splitting when it complete the count of sliding pole.

The effectiveness of the proposed protection method has been studied on a system consising of three-machine infinite bus using the PSCAD software simulation tool. The simulation tests unstable and stable oscillation, as well as loss of excitation and short-circuit fault. The simulation results show that the proposed protection method can quickly detect out-of-step conditions and is easy to be applied. The simulations on three-machine infinite bus system also prove that the proposed protection is effective for straightforward application to multi-machine systems with no need for system reduction.

\section{Proposed protection}

According to the variation of apparent impedance locus at machine terminal in out-of-step condition, the three-impedance element model uses three different impedance elements to make the out-of-step decision. The model consists of a lens impedance element and two straight-lined impedance elements and the protection characteristics are shown in the Fig.1.

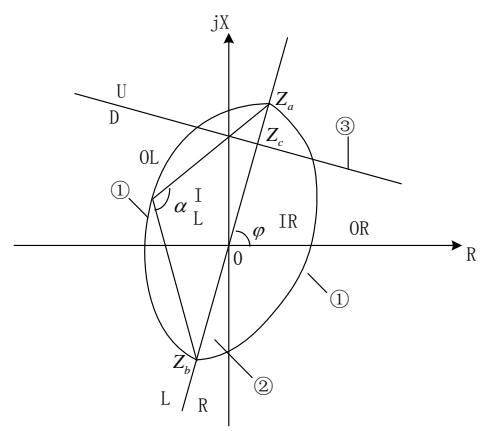

Fig. 1 three-impedance element model

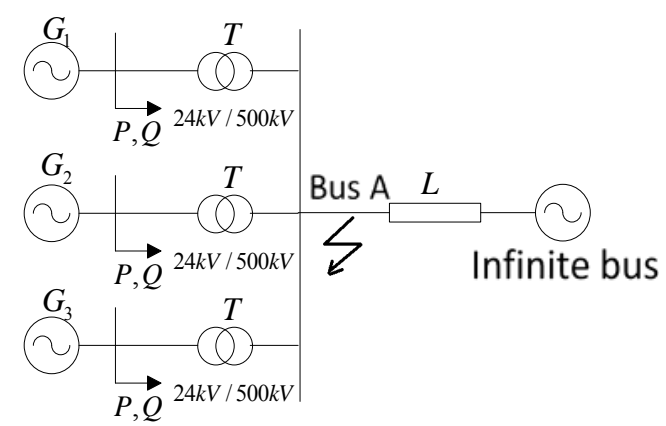

Fig. 2 three-machine infinite bus system

In Fig. 1, the lens impedance (1) divides the impedance plan into two regions. Inside the lens is active region $I$ and outside the lens is inactive region $O$. The interior angle of the lens is $\alpha$ and origin of coordinate represents where install the protection, which means the machine terminal. The beeline (2) is an occlusion straight-lined impedance element, which divides the impedance plane into L region and $\mathrm{R}$ region. When the measured impedance $\mathrm{Z}$ at machine terminal is on this line, it means . When it comes to unstable oscillation, the measured impedance $Z$ goes across the line (2), which means $\alpha$ and the generator is out of step [8]. The beeline (3) is a reactance straight-lined impedance element, which divide the impedance into $\mathrm{D}$ region and $U$ region. When the oscillation center locates in $\mathrm{D}$ region, it means the oscillation center is inside the generator-transformer unit. When the oscillation center locates in $U$ region, it means the oscillation center is outside the generator-transformer unit [9].

In this protection, EAC concept in $\delta$ domain is modified to time domain. An out-of-step detection algorithm is developed, which is based on the area under the $P-t$ curve. The proposed algorithm is based on $P-t$ curve and this information can be obtained directly from the measurements at 
substation. Fig. 3 shows the $P-\delta$ curves for stable system and the corresponding $P-t$ curve is shown in Fig.4.

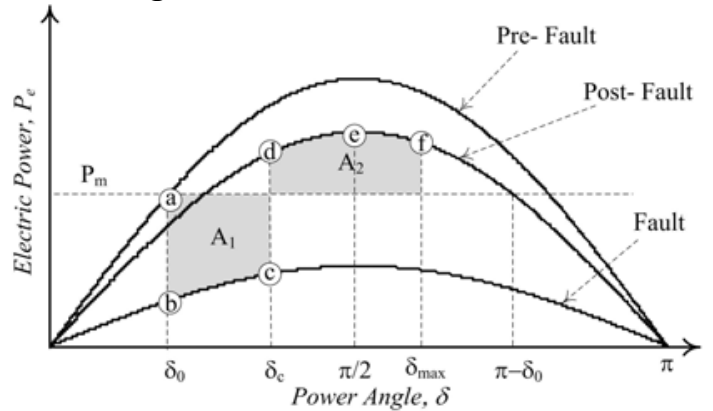

Fig. $3 \mathrm{P}-\delta$ curves illustrating a stable case

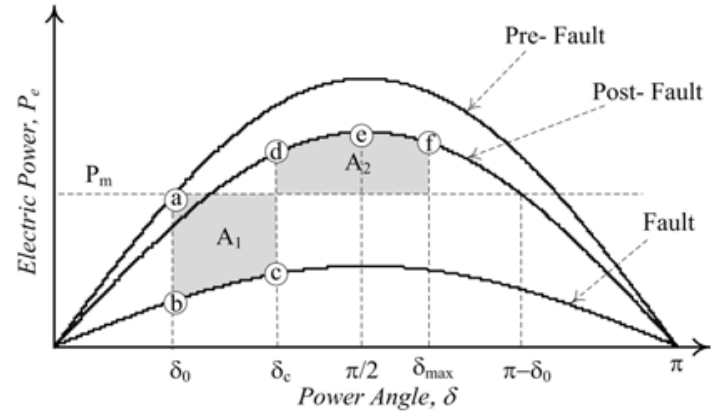

Fig.4 P-t curve for a stable case.

On the basis of energy principle, we can calculate the area of $A_{1}$ and $A_{2}$ in Fig.3. In Fig.3 and 4, $\delta_{0}$ represents power angle before the fault, $\delta_{c}$ represents power angle at the instant of fault clearing and $\delta_{\max }$ represents the maximum swing of the power angle. By applying the equal area criterion, we can judge whether a stable or an unstable oscillation occurs in the power system. When it comes to a stable oscillation, area $A_{1}$ is equal to area $A_{2}$ and area $A_{2}$ occurs before $\pi-\delta_{0}$. When it comes to an unstable oscillation, area $A_{1}$ is greater than area $A_{2}$ and area $A_{2}$ occurs at $\pi$ - $\delta_{0}$. The maximum swing of $\delta=\delta_{\max }$ for a stable swing is less than $\pi-\delta_{0}[10]$.

By applying the EAC, for a stable system, at tmax, the speed of the rotor is synchronous speed, so the speed deviation is zero. For an out-of-step condition, the speed of the rotor at tmax is greater than the synchronous speed. Thus, by summing $A_{1}$ and $A_{2}$, we can have out-of-step judgment:

For a stable condition

$$
A=A_{1}+A_{2}=\int_{t_{0}}^{t_{\max }}\left(P_{T^{*}}-P_{e^{*}}\right) d t=0
$$

For an out-of-step condition

$$
A=A_{1}+A_{2}=\int_{t_{0}}^{t_{\max }}\left(P_{T^{*}}-P_{e^{*}}\right) d t>0
$$

Equations (1) and (2) are the expressions for EAC in time domain, which tells that during the transient, if area $A_{1}$ and $A_{2}$ are equal, the system becomes stable. But if area $A_{1}$ becomes greater than area $A_{2}$, the system goes to an out-of-step condition. This concept can be referred to as the energy equilibrium criterion in the time domain. A balance of transient energy results in a stable swing whereas an unbalance of transient energy results in an out-of-step swing.

The combination of the three-impedance element model with the equal area criterion constitutes the proposed protection. For one thing, the out-of-step protection based on EAC targets at signals, predicting the potential out-of-step conditions and taking suppression actions. For another, the three-impedance element model targets at tripping and splitting when it complete the count of sliding pole. This section shows the principle of the two protection and the following sections give the simulation using the proposed technique.

\section{Software simulations}

The proposed protection has been tested on a three-machine infinite bus system and different faults are simulated by PSCAD. A three-machine infinite bus system as shown in Fig. 2 is considered to illustrate the effectiveness of the proposed technique for a multi-machine system. The parameters of the power system are given in the Appendix.

The pre-fault power angle is set at 50 degree.PSCAD simulation tool is chosen for the simulation with a simulation time step of $50 \mu$ s [11]. The number of sliding poles is set to 2 . Four different simulations are carried out as follows. 
A.Unstable oscillation. To achieve an unstable oscillation, a three-phase short-circuit fault is applied at bus $\mathrm{A}$ at $1 \mathrm{~s}$ with fault duration of $0.5 \mathrm{~s}$.

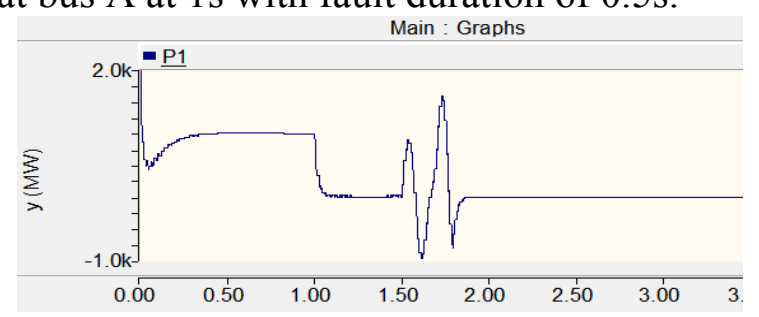

Fig.5 P-t curve of Gen 1 during an unstable oscillation

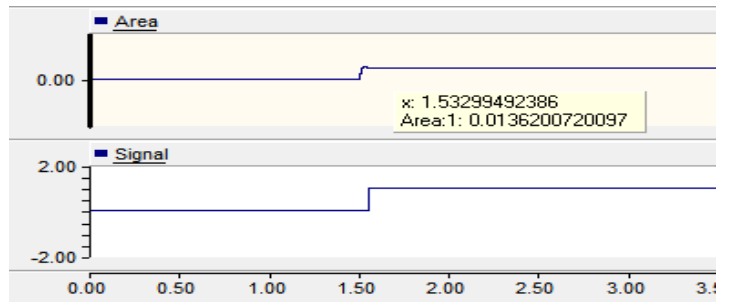

Fig.6 Area and signal curves based on EAC of Gen1 during an unstable oscillation

As we can see in Fig.5, the system goes into an unstable oscillation when the fault is cleared at 1.5s. After completing the count of sliding pole, three-impedance element model acts and cuts off Gen1 at $1.871 \mathrm{~s}$. It's worth noting that in Fig.6, area A is greater than zero $(0.0136)$ at $1.533 \mathrm{~s}$ and so an out-of-step prediction signal is made, which is earlier than the action made by three-impedance element model $(1.875 \mathrm{~s})$.

B. Stable oscillation. To achieve a stable oscillation, a three-phase short-circuit fault is applied at bus $\mathrm{A}$ at $1 \mathrm{~s}$ with fault duration of $0.1 \mathrm{~s}$.

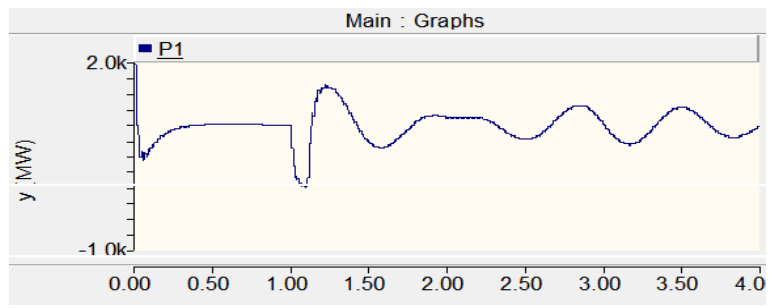

Fig.7 P-t curve of Gen1 during a stable oscillation

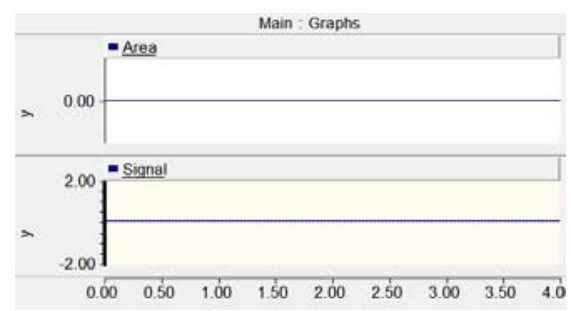

Fig.8 Area and signal curves base on EAC of Gen1 during astable oscillation

The curves in Fig.7 illustrates that during a stable oscillation, the three-impedance element model does not respond. In Fig.8, the area A remains zero. Thus, there is no signal of out-of-step prediction. C. Loss of excitation. To achieve a loss of excitation condition, the excitation system is cut off at $1.5 \mathrm{~s}$ and the loss of excitation protection does not work.

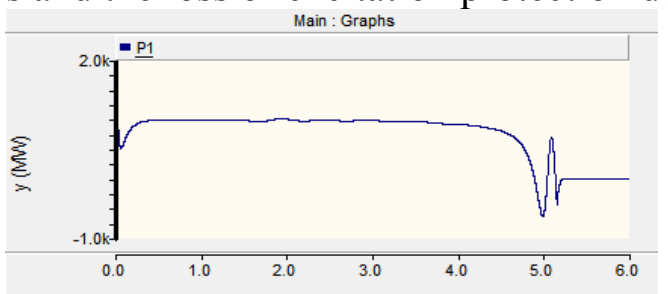

Fig.9 P-t curve of Gen1 in a loss of excitation condition of Gen 1

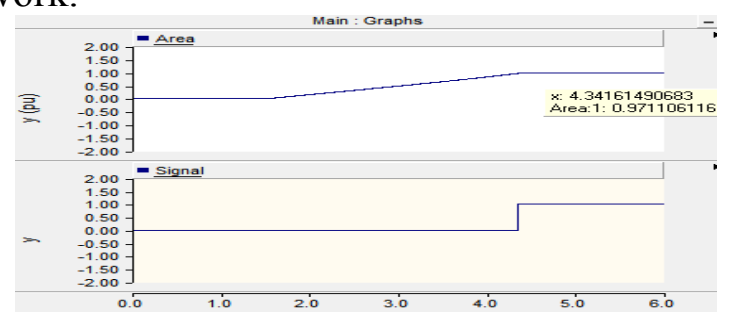

Fig.10 Area and signal curves base on EAC in a loss of excitation condition

As is shown in Fig.9, when experiencing a loss of excitation, P descends slowly and $\delta$ ascends slowly. Finally, the system goes into an unstable oscillation at $4.764 \mathrm{~s}$ and the three-impedance element model acts, cutting off Gen 1 at $5.435 \mathrm{~s}$ when it completes the count of sliding pole. Similarly, in Fig.10, the protection based on the EAC sends the out-of-step prediction signal earlier than generator tripping again at $4.342 \mathrm{~s}$.

D. Short-circuit fault. To achieve a short-circuit condition, a single-phase grounding fault is applied at bus $\mathrm{A}$ at $1 \mathrm{~s}$ and the fault remains. 


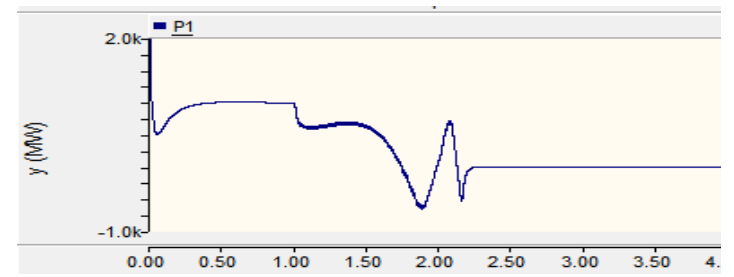

Fig. $11 \delta$-t curve of Gen1 in a loss of excitation condition

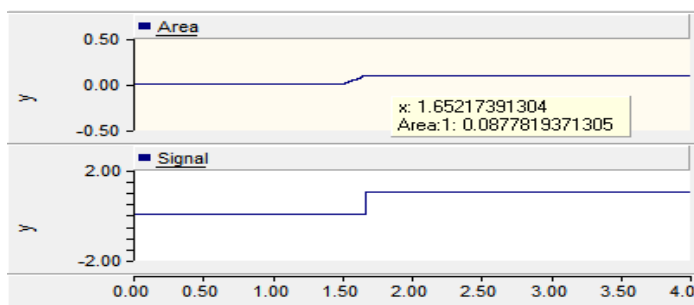

Fig.12 Area and signal curves base on EAC of Gen1 in a short-circuit condition

The curves in Fig. 11 shows that both Pe and $\delta$ fluctuate at $1 \mathrm{~s}$ and then system goes into an unstable oscillation. After completing the count of sliding pole, the three-impedance element model works and cuts off Gen 1 at 2.247s. Likewise, in Fig. 12 the area A is 0.0878 at 1.652s and the protection based on the EAC sends a prediction signal of out-of-step which is earlier than the disconnection of Gen1. What's more, from the simulation $\mathrm{C}$ and $\mathrm{D}$, we can concede that when loss of excitation and short-circuit are not promptly removed, the proposed protection can work as their backup protection.

\section{Conclusion}

This paper proposes a new procedure for out-of-step protection by combining the three-impedance element model with the equal area criterion. Taking advantage of the equal area criterion, the proposed protection can predict the out-of-step condition earlier. This type of out-of-step protection works using signals, predicting the potential out-of-step conditions and taking suppressive actions so that the stability of the system can be maintained, while the three-impedance element model simultaneously works by tripping and splitting after it has completed the count of sliding pole.

The three-machine infinite bus configuration simulation studies showed the proposed algorithm can be directly applied to a multi-machine system, without any need for a reduction of the system. Results of the simulation show the out-of-step protection can predict unstable oscillation by sending signals in the first unstable oscillation period. Meanwhile, it counts the number of sliding pole to achieve a delay action. If the loss of excitation and short-circuit faults are not promptly removed, the protection works as their backup protection. During stable oscillation, there is no need for the protection and so it does not respond. The simulation results confirm the validity of the approach presented and provide a reliable basis for out-of-step protection scheme and design of the power plant.

\section{Acknowledgements}

I would like to express my gratitude to all those who helped me during the writing of this thesis. I gratefully acknowledge the help of my supervisor, Dr. Baohua Wang, who has offered me valuable suggestions in the academic studies. In the preparation of the thesis, he has spent much time reading through each draft and provided me with inspiring advice. Without his patient instruction, insightful criticism and expert guidance, the completion of this thesis would not have been possible.

\section{References}

[1] D. Tziouvaras and D. Hou, "Out-of-step protection fundamentals and advancements," presented at the 30th Annu. Western Protective Relay Conf., Spokane, WA, Oct. 21-23, 2003.

[2] A. Y. Abdelaziz, "Adaptive protection strategies for detecting power system out-of-step conditions using neural networks," in Proc. Inst. Elect. Eng., Gen., Transm. Distrib. 1998, vol. 145, pp. 387-394.

[3] W. Rebizant, "Fuzzy logic application to out-of-step protection of generators," in Proc. IEEE Power Eng. Soc. Summer Meeting, 2001, vol. 2, pp. 927-932. 
[4] R. Padiyar and S. Krishna, "Online detection of loss of synchronism using energy function criterion,” IEEE Trans. Power Del., vol. 21, no.1, pp. 46-55, Jan. 2006.

[5] D. Q. Bi, X. A. ZHANG, "Improved out-of-step protection for synchronous generator based on the three-impedance element principle" Journal of Tsinghua University. vol.42, no.9, pp.1144-1147.2002.

[6] V. Centeno, “An adaptive out-of-step relay [for power system protection],"IEEE Trans. Power Del., vol. 12, no. 1, pp. 61-71, Jan. 1997.

[7] S. Cheng and M. S. Sachdev, "Out-of-step protection using the equal area criterion," in Proc. Canadian Conf. Electrical and Computer Engineering, 2005, pp. 1488-1491.

[8] M. A. Pai, Power System Stability: Analysis by the Direct Method of Lyapunov. Amsterdam, the Netherlands: North-Holland, 1998.P.

[9] P. Forsyth, "Real time digital simulation for control and protection system testing," in Proc. IEEE 35th Annu. Power Electronics Specialists Conf., 2004, vol. 1, pp. 329-335.

[10]D. X. Du, "An advanced real time digital simulator based test system for protection relays," in Proc. 41st Int. Univ. Power Eng. Conf., 2006, vol. 3, pp. 851-855.

[11] PSCAD User Manuals Manitoba HVDC Research Centre. Winnipeg, MB, Canada. 